was excited at relatively low potentials between 2 and 10 kilovolts.

Other work with soft X-rays produced by an ordinary tube is shown by $\mathbf{H}$. Flower and Messrs. Ilford Ltd. The structures of shells and flowers are thus illustrated.

From the very excellent Natural History Section mention may be made of an interesting series of photographs by H. Morrey Salmon illustrating the habits of the Manx shearwater. This bird comes to land only during the breeding season, when the colonies sometimes number thousands of pairs. On shore the birds move only by night. They nest in burrows which they share with rabbits. The nesting bird is said to remain underground during the hours of daylight, and is relieved by its mate at night. Some of the photographs were taken off Pembrokeshire at about 10.45 p.m. about midsummer at a distance of nearly two miles; others, taken by flashlight, were taken close to the birds.

An instructional exhibit by Messrs. Ilford Ltd. shows a 'working model' of the formation of halation circles on plates and films. Excellent examples of the influence of various backings in preventing halation are shown. Further exhibits by Ilford Ltd. in cooperation with Dr. Russell Reynolds show examples of cineradiographs of such subjects as the beating heart, moving joints, etc. The Cossor-Robertson cardiograph is demonstrated by Messrs. A. C. Cossor Ltd. and Ilford Ltd., together with typical cardiograms made on special photographic material manufactured by the latter firm.

\section{Magnetic Materials}

$\mathrm{F}$ OR some years past it has been the practice of line communication engineers to make use of iron-cored inductance coils and transformers at speech frequencies and even at carrier frequencies up to 50,000 cycles per second. The cores of such coils were usually composed of iron or a magnetic alloy in the form of wire or of powder embedded in a suitable binding material. During the past year or so, the attention of those responsible for the design and production of wireless receivers has been directed to the possibility of using such magnetic cores in coils and transformers, the frequencies of operation of which may exceed one million cycles per second. It has been claimed that broadcasting receivers giving better performance in a smaller space can be designed by the use of such coils, on account of the facility in screening and the more effective coupling between the circuits which these coils afford.

A report*, recently published by the Radio Research Board, summarises existing knowledge, both theoretical and experimental, of the behaviour of magnetic materials at radio frequencies with the object of assisting those engaged in the application of these materials in the manner outlined above. The subject is surveyed in an approximately analytical manner intended to illustrate the individual proper. ties, such as permeability, hysteresis and dielectric loss of the various materials examined; and, where the quantitative data permit, typical numerical values are assigned to these properties for various

* Department of Scientific and Industrial Research. Radio Research. Special Report No. 14. "Magnetic Materials at Radio Frequencies, A Critical Survey of Present Knowledge." By F. M. Colebrook, (London: H.M. Stationery Office, 1934.) 6d. net.

\section{at Radio Frequencies}

frequencies up to about 2,000 kilocycles per second. It appears that these electrical properties of ferro. magnetic core materials may be expressed in terms of permeability and power-factor in a manner analogous to the use of permittivity and power. factor for dielectrics.

From the information summarised in this report, it is concluded that the effective permeability of magnetic powder compositions is necessarily low compared with that of the magnetic material, and that it depends mainly upon the size of the particles employed and the spacing between them, rather than upon the permeability of the magnetic constituent. It is probable, though not certain, that the greater part of the total radio frequency losses in composite magnetic materials is due to eddy-current formation and a consequent skin-effect in flux distribution. There are at present available a number of iron powder compositions in which, by sufficiently fine division of the magnetic material and provision of adequate particle insulation, the total losses are reduced to a value which gives an economic permeability of from 4 to 10 up to frequencies of about 2,000 kilocycles per second. It is not known to what extent the behaviour of such material is consistent with theory, nor is there any certain knowledge of the constitution of the losses. Further, it is concluded that there is no evidence indicating that the limit of improvement in such materials has yet been reached.

The useful lines of future investigation of the subject are clearly indicated in the report, which undoubtedly forms a clear and concise introduction to this field of research in radio frequency technique.

\title{
Determination of the Molecular Weights of Colloids
}

$I^{\mathrm{N}}$ the January issue of the Berichte der deutschen chemischen Gesellschaft Prof. H. Staudinger discusses the validity of the method of deducing the molecular weight of a colloid of high molecular complexity from observations of its specific viscosity. The author claims that this new method is applicable to the investigation of numerous complex naturally occur. ring substances such as cellulose and india-rubber, since many physical properties such as elasticity, ductility, solubility, etc., are functions of the length of the molecular chain. Older methods of determining molecular weights of complex molecules are more restricted in their application. Thus the cryoscopic methods give very low results in dilute solutions and in more concentrated solutions the relation between molecular weight and osmotic pressure is no longer simple. The diffusion method is only suitable for particles of spherical shape and can therefore not be applied to the long fibre-molecules of many natural colloids.

An earlier account of Prof. Staudinger's views is to be found in the Transactions of the Faraday Society of January 1933, and the main object of this recent paper is to examine certain experimental evidence 